\title{
TOTAL CRYSTAL ENERGY AND HEAT OF SOLUTION OF ALKALI BASED BINARY ALLOYS
}

\author{
P.N. GAJJAR ${ }^{a}$, B.Y. ThaKorE ${ }^{b}$ AND A.R. JANI ${ }^{a}$ \\ ${ }^{a}$ Department of Physics, Sardar Patel University \\ Vallabh Vidyanagar 388 120, India \\ ${ }^{b}$ Department of Physics, V.P. \& R.P.T.P. Science College \\ Vallabh Vidyanagar 388 120, India
}

(Received October 25, 2000; revised version January 22, 2001)

\begin{abstract}
A detailed study of the total crystal energy and heat of solution of ten alkali based alloys is made within the framework of the second order perturbation theory and employing the pseudo alloy atom model. Three different forms of the local field correction functions, viz. Harrison, Taylor, and Ichimaru and Utsumi, are used to incorporate the exchange and correlation effects while computing the band structure part of the total energy. Reasonable agreement with the experimental values of the total energy of pure components is found (corresponding to the concentration factor $x=0$ or 1). It is observed that for all the systems, for small impurity concentration factors, the alloys form very good homogeneous solid solutions. Also the interaction parameter $\Delta E / x(1-x)$ does not depend on $x$, whenever the difference in the atomic sizes of the two components making up the alloy is small.
\end{abstract}

PACS numbers: $64.10 .+\mathrm{h}, 71.15 . \mathrm{Hx}$

\section{Introduction}

It is known that the electronic properties of simple metals can be understood using the pseudopotentials. The total energy of pure metals in the framework of the second order perturbation theory has been a subject of many pseudopotential studies. The underlying assumption in the calculation of the total energy of alloys is the same as that for pure metals.

The first calculations of an alloy's band energy were made by Hayes and co-workers [1] using a non-local potential and by Inglesfield [2] using a local form of the potential. They calculated the ordering energy in $\mathrm{Li}-\mathrm{Mg}$ and $\mathrm{Hg}-\mathrm{Mg}$ systems, respectively. Also Stroud and Ashcroft [3] have studied various properties of alloys in the same second order perturbation scheme as in the case of pure metals. Krasko and Gurskii [4] have calculated the energy of a completely disordered alloy, 
characterized by a long range order. They have also calculated the concentration dependence of the lattice constant in $\mathrm{K}-\mathrm{Cs}$ and $\mathrm{K}-\mathrm{Rb}$ alloys. We have also reported the theoretical total energy and its screening dependence for various alkali based alloys $[5-10]$.

Hafner [11] derived an orthogonalized plane wave based on the first principles pseudopotential method and applied it to investigate the random binary alloys and ordered intermetallic compounds between alkali metals. This treatment is the virtual crystal approximation in which the disordered alloy is replaced by a monatomic periodic lattice and the alloy potential is considered as a linear combination of the average lattice potential and the difference potential. Tanigawa and Doyama [12] have used a different approach for the study of the total crystal energy of alloys. This approach is known as the pseudo alloy atom (PAA) model in which a hypothetical monatomic crystal is supposed to be composed of pseudo alloy atoms. In an actual alloy different alloy constituent atoms model the atoms of a single species, the pseudo alloy atoms occupy the lattice sites and form a perfect lattice in the same way as pure metals. This model takes into account the self-consistent treatment implicitly. In this model the hypothetical crystal made up of pseudo alloy atoms is supposed to have the same properties as the actual disordered alloy crystal and the pseudopotential formalism is then applied to calculate various properties of an alloy.

Looking at the above stated limited study of alloys we thought worthwhile to use the PAA model in the present investigation to compute the total crystal energy and heat of solutions of ten alkali based alloys, viz. Li-Na, Li-K, Li-Rb, $\mathrm{Li}-\mathrm{Cs}, \mathrm{Na}-\mathrm{K}, \mathrm{Na}-\mathrm{Rb}, \mathrm{Na}-\mathrm{Cs}, \mathrm{K}-\mathrm{Rb}, \mathrm{K}-\mathrm{Cs}$, and $\mathrm{Rb}-\mathrm{Cs}$ systems. To describe electron-ion interactions in the binary systems, the bare ion local model potential employed in the present investigation is given as $[13,14]$ :

$$
W_{\mathrm{B}}(r)= \begin{cases}0, & r<r_{\mathrm{c}} \\ -2 Z / r\left[1-\exp \left(-r / r_{\mathrm{c}}\right)\right], & r \geq r_{\mathrm{c}}\end{cases}
$$

The Fourier transform of the potential into $\boldsymbol{q}$-space is given by $[13,14]$

$$
W_{\mathrm{B}}(q)=-\frac{8 \pi Z}{\Omega_{0} q^{2}}\left\{\cos \left(q r_{c}\right)-\frac{q r_{\mathrm{c}} \exp (-1)}{\left(1+q^{2} r_{\mathrm{c}}^{2}\right)}\left[\sin \left(q r_{\mathrm{c}}\right)+q r_{\mathrm{c}} \cos \left(q r_{\mathrm{c}}\right)\right]\right\}
$$

This potential contains a single parameter $r_{c}$. Instead of finding the potential parameter by fitting a physical property or from the first zero of the form factor, we have applied the zero pressure condition for the determination of the parameter. This model potential has previously produced very good results about susceptibility and electrical transport properties of simple metals $[13,14]$.

\section{Theory}

In the PAA model the alloy can be thought of as composed of a system of $N$ periodically arranged positive ions immersed in an electron gas. The alloy of the type $A_{1-x} B_{x}$ is made up of two species $\mathrm{A}$ and $\mathrm{B}$ with an arbitrary concentration $x$. 
The total crystal energies per pseudo-atom, $E(x)$, of the binary alloy can be obtained in the framework of the usual second order perturbation as $[5-10,15-18]$

$$
E(x)=E_{i}(x)+E_{e s}(x)+E_{1}(x)+E_{2}(x),
$$

where the individual energy terms are given by

$$
E_{i}(x)=-\alpha Z_{\text {alloy }}^{2} / R_{\mathrm{a}},
$$

where $R_{\mathrm{a}}=r_{\mathrm{s}} Z_{\text {alloy }}^{1 / 3}$; here $r_{\mathrm{s}}$ is the radius of the sphere containing one electron and $\alpha$ is Madelung's constant;

$$
\begin{aligned}
& E_{e s}(x)=Z_{\text {alloy }}\left[2.21 / r_{\mathrm{s}}^{2}-0.916 / r_{\mathrm{s}}-0.115+0.031 \ln \left(r_{\mathrm{s}}\right)\right], \\
& E_{1}(x)=\lim _{q \rightarrow 0}\left[4 \pi Z_{\text {alloy }}^{2} e^{2} / \Omega_{0, \text { alloy }} q^{2}+Z_{\text {alloy }} W_{\mathrm{B}}(q)\right]
\end{aligned}
$$

which in the case of our local model potential has the form $[17,18]$

$$
\begin{aligned}
& E_{1}(x)=4 \pi Z^{2} r_{c, \text { alloy }}^{2} / \Omega_{0, \text { alloy }}[1+\exp (-1)], \\
& E_{2}(x)=\sum_{q \neq 0} F(q)
\end{aligned}
$$

is the band structure energy, here $F(q)$ - the energy wave number characteristics given as

$$
F(q)=-\frac{\Omega_{0, \text { alloy }}}{16 \pi} q^{2}\left|W_{\mathrm{B}}(q)\right|^{2} \frac{\left[\varepsilon_{\mathrm{H}}(q)-1\right]}{\left\{1+\left[\varepsilon_{\mathrm{H}}(q)-1\right][1-f(q)]\right\}} .
$$

The values of atomic volume $\Omega_{0}$ and potential parameter $r_{\mathrm{c}}$ for the alloy $\mathrm{A}_{1-x} \mathrm{~B}_{x}$ can be found from the relation

$$
\begin{aligned}
& \Omega_{0, \text { alloy }}=(1-x) \Omega_{\mathrm{A}}+x \Omega_{\mathrm{B}}, \\
& r_{\mathrm{c}, \text { alloy }}=(1-x) r_{\mathrm{cA}}+x r_{\mathrm{cB}},
\end{aligned}
$$

where $\Omega_{\mathrm{A}}$ and $\Omega_{\mathrm{B}}$ are the atomic volumes of the two pure components. $r_{\mathrm{cA}}$ and $r_{\mathrm{CB}}$ are the parameters of the potential for the two pure components found by satisfying the zero pressure condition.

Next we consider the phase mixture of $\mathrm{A}_{1-x}+B_{x}$ and express the crystal energy of the mixture as following expression:

$$
E_{\text {mix }}(x)=(1-x) E_{\mathrm{A}}+x E_{\mathrm{B}} .
$$

Here $E_{\mathrm{A}}$ and $E_{\mathrm{B}}$ are the total crystal energies of the two pure components forming an alloy. The total crystal energy and the energy of mixing are then used to find the heat of solution via the relation

$$
\Delta E(x)=E(x)-E_{\operatorname{mix}}(x) .
$$




\section{Results and discussion}

The constants and parameters used in the present investigation are tabulated in Table I. The total crystal energy of alkali based alloys found with our proposed model potential using Eqs. (1) to (12) are tabulated in Tables II to XI. We have used three different forms of the local field correction functions, viz. Harrison (H) [19], Taylor (T) [20], and Ichimaru-Utsumi (IU) [21], to incorporate the exchange and correlation effects while computing the band structure part of the total energy. In these tables the value of the concentration factor $x$ corresponding to a value of 0 or 1 will give the total energy of the pure components making up the

\section{TABLE I}

The constants and parameters used in the present investigation.

\begin{tabular}{c|c|c|c|c|c|c|c}
\hline \hline Metal & \multirow{2}{*}{$\Omega_{0}$} & $k_{\mathrm{F}}$ & $r_{\mathrm{s}}$ & \multicolumn{3}{|c|}{$r_{\mathrm{c}}$ (a.u.) } & \begin{tabular}{c}
$-E_{\text {tot }}$ (Expt.) \\
\cline { 5 - 7 }
\end{tabular} \\
\cline { 5 - 7 } (a.u.) & (a.u.) & (a.u.) & $\mathrm{H}$ & $\mathrm{T}$ & $\mathrm{IU}$ & 0.5162 \\
$\mathrm{Li}$ & 144.9 & 0.5890 & 3.258 & 0.8142 & 0.8078 & 0.8104 & $0.82,23]$ \\
$\mathrm{Na}$ & 254.5 & 0.4882 & 3.931 & 1.0786 & 1.0729 & 1.0752 & 0.4598 \\
$\mathrm{~K}$ & 481.4 & 0.3947 & 4.862 & 1.4433 & 1.4408 & 1.4417 & 0.3878 \\
$\mathrm{Rb}$ & 587.9 & 0.3693 & 5.197 & 1.5752 & 1.5747 & 1.5747 & 0.3700 \\
$\mathrm{Cs}$ & 745.5 & 0.3412 & 5.625 & 1.7447 & 1.7474 & 1.7406 & 0.3451
\end{tabular}

TABLE II

Total crystal energy, $E_{\text {Tot }}$ (Ryd.), for the $\mathrm{Li}_{1-x} \mathrm{Na}_{x}$ alloy.

\begin{tabular}{l|c|c|c}
\hline \hline \multicolumn{1}{c|}{$x$} & $\mathrm{H}$ & $\mathrm{T}$ & $\mathrm{IU}$ \\
\hline $0.0(\mathrm{Li})$ & 0.56416 & 0.56723 & 0.56594 \\
0.1 & 0.55444 & 0.55737 & 0.55614 \\
0.2 & 0.54504 & 0.54783 & 0.54665 \\
0.3 & 0.53595 & 0.53860 & 0.53748 \\
0.4 & 0.52716 & 0.52926 & 0.52862 \\
0.5 & 0.51866 & 0.52108 & 0.52005 \\
0.6 & 0.51044 & 0.51274 & 0.51175 \\
0.7 & 0.50247 & 0.50467 & 0.50373 \\
0.8 & 0.49476 & 0.49686 & 0.49595 \\
0.9 & 0.48727 & 0.48928 & 0.48841 \\
$1.0(\mathrm{Na})$ & 0.48001 & 0.48193 & 0.48109
\end{tabular}


alloys. The experimental total crystal energy of these alloys are not available in the literature hence we have compared presently obtained values at the concentration $x=0$ and 1 with the experimental values of the pure components. It is observed from the tables that those values agree reasonably well with the experimental data $[22,23]$.

TABLE III

Total crystal energy, $E_{\text {Tot }}$ (Ryd.), for the $\mathrm{Li}_{1-x} \mathrm{~K}_{x}$ alloy.

\begin{tabular}{l|c|c|c}
\hline \hline \multicolumn{1}{c|}{$x$} & $\mathrm{H}$ & $\mathrm{T}$ & $\mathrm{IU}$ \\
\hline $0.0(\mathrm{Li})$ & 0.56416 & 0.56723 & 0.56594 \\
0.1 & 0.54132 & 0.54399 & 0.54287 \\
0.2 & 0.52013 & 0.52247 & 0.52148 \\
0.3 & 0.50065 & 0.50268 & 0.50181 \\
0.4 & 0.48271 & 0.48449 & 0.48372 \\
0.5 & 0.46615 & 0.46768 & 0.46701 \\
0.6 & 0.45007 & 0.45210 & 0.45150 \\
0.7 & 0.43643 & 0.43757 & 0.43705 \\
0.8 & 0.42299 & 0.42398 & 0.42351 \\
0.9 & 0.41035 & 0.41120 & 0.41078 \\
$1.0(\mathrm{~K})$ & 0.39841 & 0.39915 & 0.39877
\end{tabular}

TABLE IV

Total crystal energy, $E_{\text {Tot }}$ (Ryd.), for the $\mathrm{Li}_{1-x} \mathrm{Rb} \mathrm{b}_{x}$ alloy.

\begin{tabular}{l|c|c|c}
\hline \hline \multicolumn{1}{c|}{$x$} & $\mathrm{H}$ & $\mathrm{T}$ & $\mathrm{IU}$ \\
\hline $0.0(\mathrm{Li})$ & 0.56416 & 0.56723 & 0.56594 \\
0.1 & 0.53654 & 0.53911 & 0.53803 \\
0.2 & 0.51132 & 0.51349 & 0.51257 \\
0.3 & 0.48861 & 0.49042 & 0.48964 \\
0.4 & 0.46809 & 0.46960 & 0.46894 \\
0.5 & 0.44994 & 0.45067 & 0.45012 \\
0.6 & 0.43235 & 0.43334 & 0.43289 \\
0.7 & 0.41659 & 0.41738 & 0.41700 \\
0.8 & 0.40196 & 0.40258 & 0.40226 \\
0.9 & 0.38831 & 0.38879 & 0.38852 \\
$1.0(\mathrm{Rb})$ & 0.37549 & 0.37587 & 0.37563
\end{tabular}


The heat of solution for all the ten alkali based alloys are shown in Figs. 1 to 10 . The ratio $\Delta E / x(1-x)$ has also been plotted for them on the same sheet. A study of these graphs shows that for all the ten systems we have obtained small and positive values for the heat of solution in the entire concentration range.

\section{TABLE V}

Total crystal energy, $E_{\text {Tot }}$ (Ryd.), for the $\mathrm{Li}_{1-x} \mathrm{Cs}_{x}$ alloy.

\begin{tabular}{l|c|c|c}
\hline \hline \multicolumn{1}{c|}{$x$} & $\mathrm{H}$ & $\mathrm{T}$ & $\mathrm{IU}$ \\
\hline $0.0(\mathrm{Li})$ & 0.56416 & 0.56723 & 0.56594 \\
0.1 & 0.53029 & 0.53273 & 0.53170 \\
0.2 & 0.50006 & 0.50202 & 0.50119 \\
0.3 & 0.47361 & 0.47516 & 0.47449 \\
0.4 & 0.45028 & 0.45146 & 0.45094 \\
0.5 & 0.42947 & 0.43033 & 0.42993 \\
0.6 & 0.41070 & 0.41129 & 0.41099 \\
0.7 & 0.39359 & 0.39396 & 0.39375 \\
0.8 & 0.37788 & 0.37806 & 0.37792 \\
0.9 & 0.36333 & 0.36338 & 0.36328 \\
$1.0(\mathrm{Cs})$ & 0.34976 & 0.34973 & 0.34965
\end{tabular}

TABLE VI

Total crystal energy, $E_{\text {Tot }}($ Ryd.), for the $\mathrm{Na}_{1-x} \mathrm{~K}_{x}$ alloy.

\begin{tabular}{l|c|c|c}
\hline \hline \multicolumn{1}{c|}{$x$} & $\mathrm{H}$ & $\mathrm{T}$ & $\mathrm{IU}$ \\
\hline $0.0(\mathrm{Na})$ & 0.48001 & 0.48193 & 0.48109 \\
0.1 & 0.47023 & 0.47208 & 0.47131 \\
0.2 & 0.46102 & 0.46262 & 0.46191 \\
0.3 & 0.45208 & 0.45355 & 0.45289 \\
0.4 & 0.44351 & 0.44484 & 0.44423 \\
0.5 & 0.43526 & 0.43647 & 0.43592 \\
0.6 & 0.42734 & 0.42844 & 0.42792 \\
0.7 & 0.41971 & 0.42070 & 0.42023 \\
0.8 & 0.41235 & 0.41326 & 0.41282 \\
0.9 & 0.40526 & 0.40608 & 0.40567 \\
$1.0(\mathrm{~K})$ & 0.39841 & 0.39915 & 0.39877
\end{tabular}


This has also been observed in some other theoretical investigations carried out by Tanigawa and Doyama [12], Soma et al. [15, 16], and Tewari and Khanna [17]. The positive values of energy differences predict that the homogeneous solid solution is thermodynamically unstable relative to a phase mixture at low temperature. All

\section{TABLE VII}

Total crystal energy, $E_{\text {Tot }}$ (Ryd.), for the $\mathrm{Na}_{1-x} \mathrm{Rb}_{x}$ alloy.

\begin{tabular}{l|c|c|c}
\hline \multicolumn{1}{c|}{$x$} & $\mathrm{H}$ & $\mathrm{T}$ & $\mathrm{IU}$ \\
\hline $0.0(\mathrm{Na})$ & 0.48001 & 0.48193 & 0.48109 \\
0.1 & 0.46689 & 0.46857 & 0.46783 \\
0.2 & 0.45446 & 0.45592 & 0.45527 \\
0.3 & 0.44270 & 0.44397 & 0.44339 \\
0.4 & 0.43157 & 0.43267 & 0.43216 \\
0.5 & 0.42103 & 0.42197 & 0.42152 \\
0.6 & 0.41102 & 0.41182 & 0.41143 \\
0.7 & 0.40151 & 0.40218 & 0.40184 \\
0.8 & 0.39244 & 0.39300 & 0.39270 \\
0.9 & 0.38378 & 0.38424 & 0.38398 \\
$1.0(\mathrm{Rb})$ & 0.37549 & 0.37587 & 0.37563
\end{tabular}

TABLE VIII

Total crystal energy, $E_{\text {Tot }}$ (Ryd.), for the $\mathrm{Na}_{1-x} \mathrm{Cs}_{x}$ alloy.

\begin{tabular}{l|c|c|c}
\hline \hline \multicolumn{1}{c|}{$x$} & $\mathrm{H}$ & $\mathrm{T}$ & $\mathrm{IU}$ \\
\hline $0.0(\mathrm{Na})$ & 0.48001 & 0.48193 & 0.48109 \\
0.1 & 0.46251 & 0.46408 & 0.46338 \\
0.2 & 0.44619 & 0.44747 & 0.44689 \\
0.3 & 0.43106 & 0.43208 & 0.43161 \\
0.4 & 0.41703 & 0.41782 & 0.41744 \\
0.5 & 0.40397 & 0.40457 & 0.40426 \\
0.6 & 0.39177 & 0.39220 & 0.39195 \\
0.7 & 0.38033 & 0.38061 & 0.38042 \\
0.8 & 0.36957 & 0.36972 & 0.36958 \\
0.9 & 0.35940 & 0.35945 & 0.35934 \\
$1.0(\mathrm{Cs})$ & 0.34976 & 0.34973 & 0.34965
\end{tabular}


the three screening functions exhibit the same trend for the energy difference. The numerical values of the heat of mixing are almost identical for lower and higher values of concentrations, while showing a difference which is maximum at about $x=0.5$.

TABLE IX

Total crystal energy, $E_{\text {Tot }}$ (Ryd.), for the $\mathrm{K}_{1-x} \mathrm{Rb}_{x}$ alloy.

\begin{tabular}{l|c|c|c}
\hline \hline \multicolumn{1}{c|}{$x$} & $\mathrm{H}$ & $\mathrm{T}$ & $\mathrm{IU}$ \\
\hline $0.0(\mathrm{~K})$ & 0.39841 & 0.39915 & 0.39877 \\
0.1 & 0.39598 & 0.39668 & 0.39623 \\
0.2 & 0.39359 & 0.39425 & 0.39391 \\
0.3 & 0.39123 & 0.39185 & 0.39152 \\
0.4 & 0.38890 & 0.38948 & 0.38917 \\
0.5 & 0.38659 & 0.38714 & 0.38684 \\
0.6 & 0.38432 & 0.38483 & 0.38454 \\
0.7 & 0.38207 & 0.38255 & 0.38227 \\
0.8 & 0.37985 & 0.38029 & 0.38003 \\
0.9 & 0.37766 & 0.37806 & 0.37782 \\
$1.0(\mathrm{Rb})$ & 0.37549 & 0.37587 & 0.37563
\end{tabular}

TABLE X

Total crystal energy, $E_{\text {Tot }}$ (Ryd.), for the $\mathrm{K}_{1-x} \mathrm{Cs}_{x}$ alloy.

\begin{tabular}{l|c|c|c}
\hline \hline \multicolumn{1}{c|}{$x$} & $\mathrm{H}$ & $\mathrm{T}$ & $\mathrm{IU}$ \\
\hline $0.0(\mathrm{~K})$ & 0.39841 & 0.39915 & 0.39877 \\
0.1 & 0.39291 & 0.39355 & 0.39321 \\
0.2 & 0.38758 & 0.38812 & 0.38782 \\
0.3 & 0.38239 & 0.38283 & 0.38257 \\
0.4 & 0.37734 & 0.37770 & 0.37747 \\
0.5 & 0.37243 & 0.37272 & 0.37252 \\
0.6 & 0.36766 & 0.36787 & 0.36770 \\
0.7 & 0.36301 & 0.36315 & 0.36301 \\
0.8 & 0.35848 & 0.35856 & 0.35844 \\
0.9 & 0.35407 & 0.35408 & 0.35399 \\
$1.0(\mathrm{Cs})$ & 0.34976 & 0.34973 & 0.34965
\end{tabular}


TABLE XI

Total crystal energy, $E_{\text {Tot }}$ (Ryd.), for the $\mathrm{Rb}_{1-x} \mathrm{Cs}_{x}$ alloy.

\begin{tabular}{l|c|c|c}
\hline \hline \multicolumn{1}{c|}{$x$} & $\mathrm{H}$ & $\mathrm{T}$ & $\mathrm{IU}$ \\
\hline $0.0(\mathrm{Rb})$ & 0.37549 & 0.37587 & 0.37563 \\
0.1 & 0.37274 & 0.37307 & 0.37285 \\
0.2 & 0.37003 & 0.37031 & 0.37012 \\
0.3 & 0.36737 & 0.36760 & 0.36742 \\
0.4 & 0.36474 & 0.36493 & 0.36477 \\
0.5 & 0.36215 & 0.36230 & 0.36215 \\
0.6 & 0.35960 & 0.35971 & 0.35958 \\
0.7 & 0.35709 & 0.35716 & 0.35704 \\
0.8 & 0.35461 & 0.35465 & 0.35454 \\
0.9 & 0.35217 & 0.35217 & 0.35208 \\
$1.0(\mathrm{Cs})$ & 0.34976 & 0.34973 & 0.34965
\end{tabular}

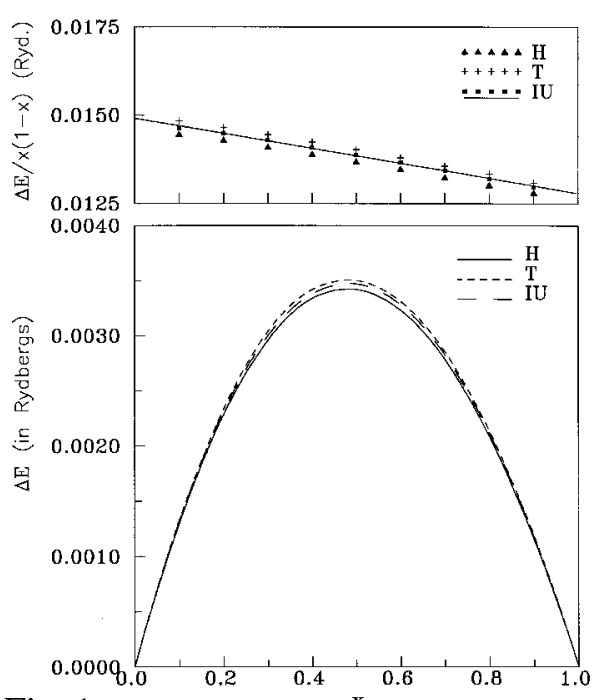

Fig. 1

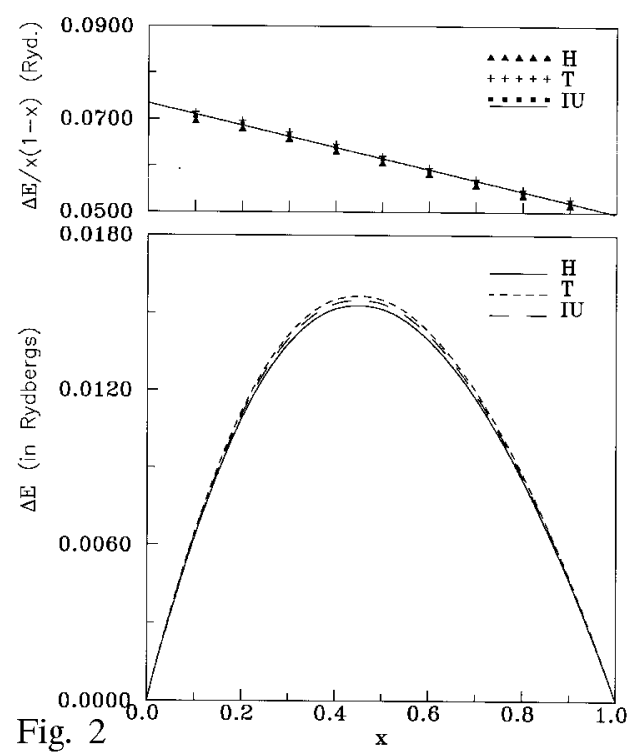

Fig. 2

Fig. 1. The energy difference and interaction parameter for $\mathrm{Li}-\mathrm{Na}$ alloy.

Fig. 2. The energy difference and interaction parameter for $\mathrm{Li}-\mathrm{K}$ alloy. 

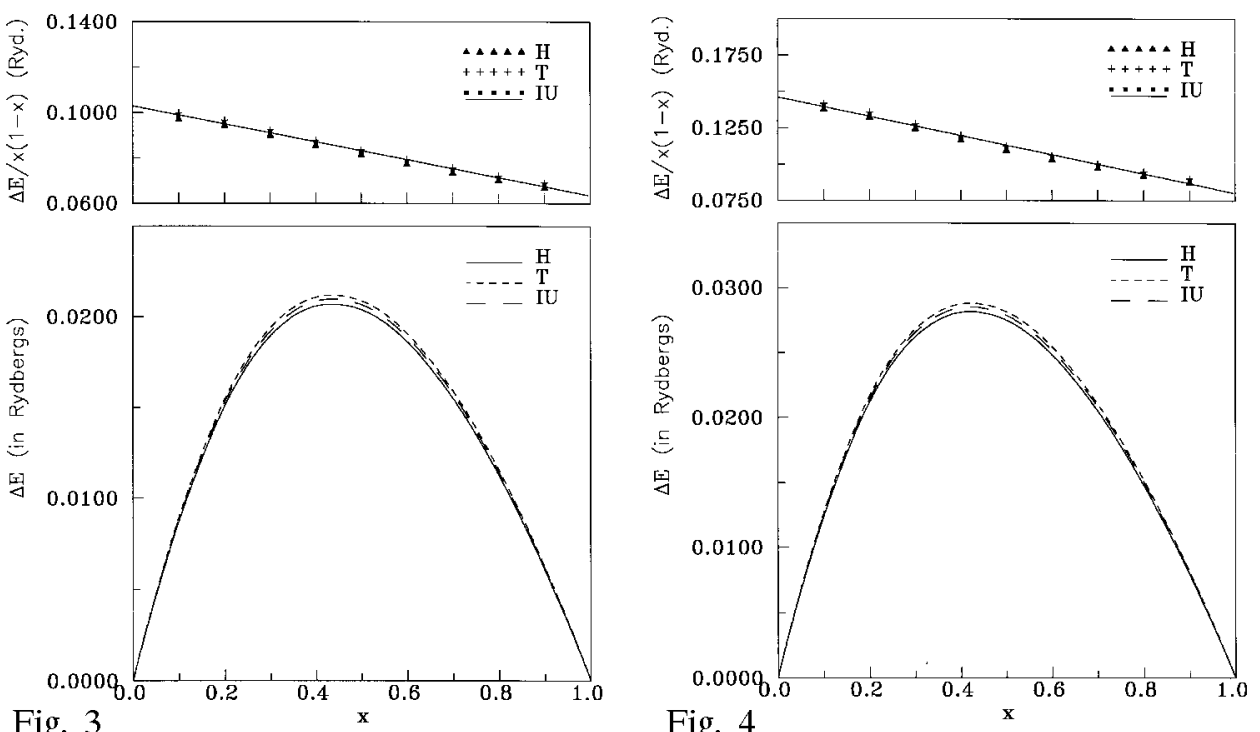

Fig. 4

Fig. 3. The energy difference and interaction parameter for $\mathrm{Li}-\mathrm{Rb}$ alloy.

Fig. 4. The energy difference and interaction parameter for $\mathrm{Li}-\mathrm{Cs}$ alloy.
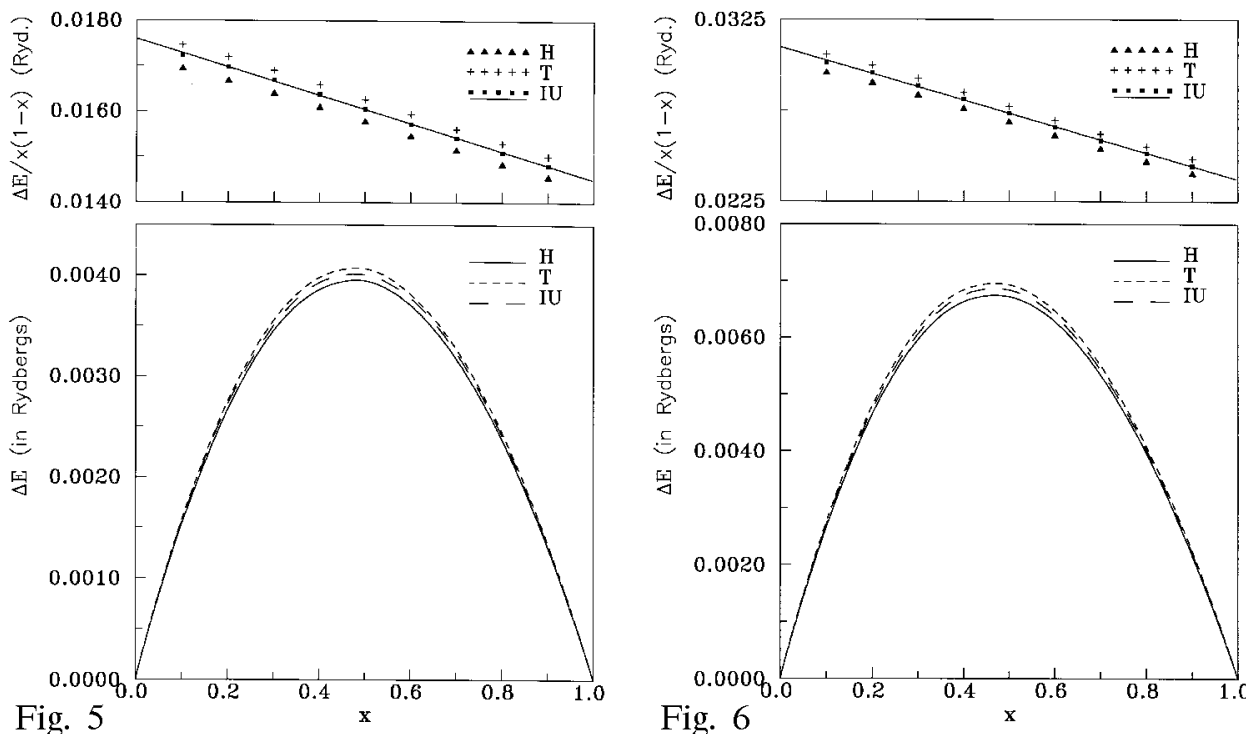

Fig. 5. The energy difference and interaction parameter for $\mathrm{Na}-\mathrm{K}$ alloy.

Fig. 6. The energy difference and interaction parameter for $\mathrm{Na}-\mathrm{Rb}$ alloy. 

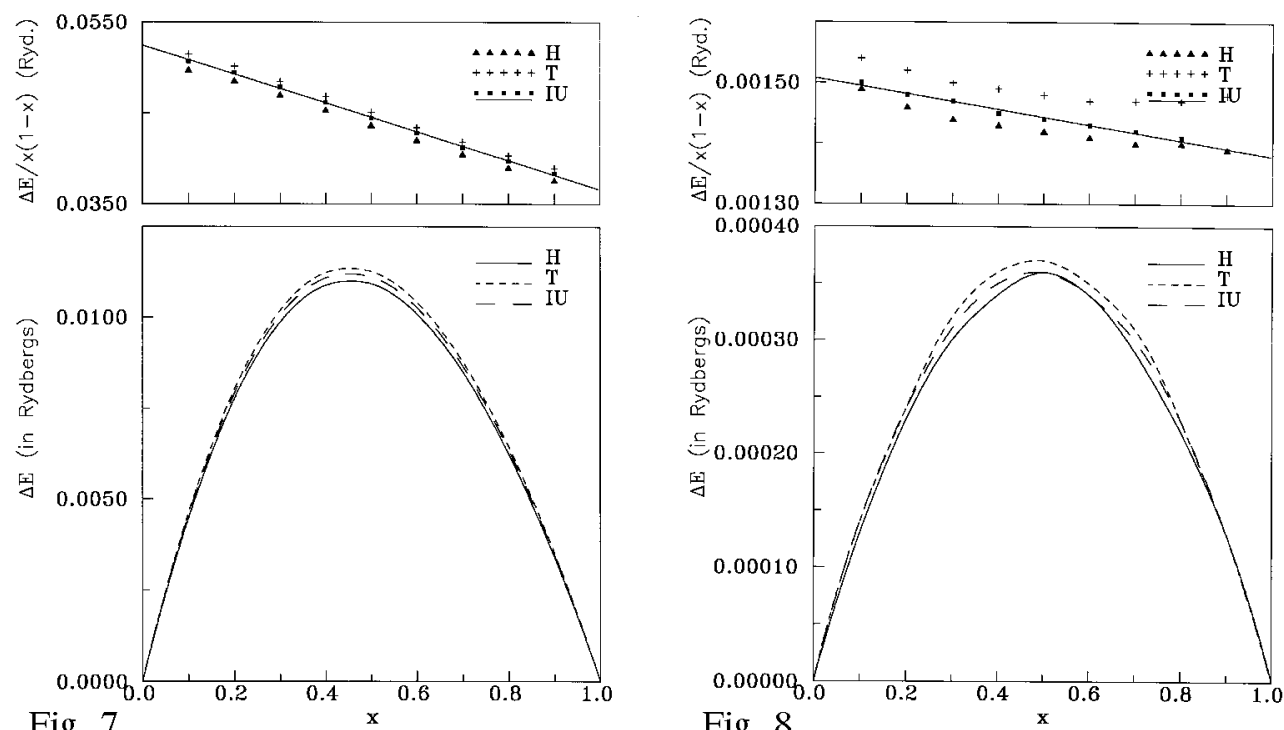

Fig. 7

Fig. 8

Fig. 7. The energy difference and interaction parameter for $\mathrm{Na}-\mathrm{Cs}$ alloy.

Fig. 8. The energy difference and interaction parameter for $\mathrm{K}-\mathrm{Rb}$ alloy.
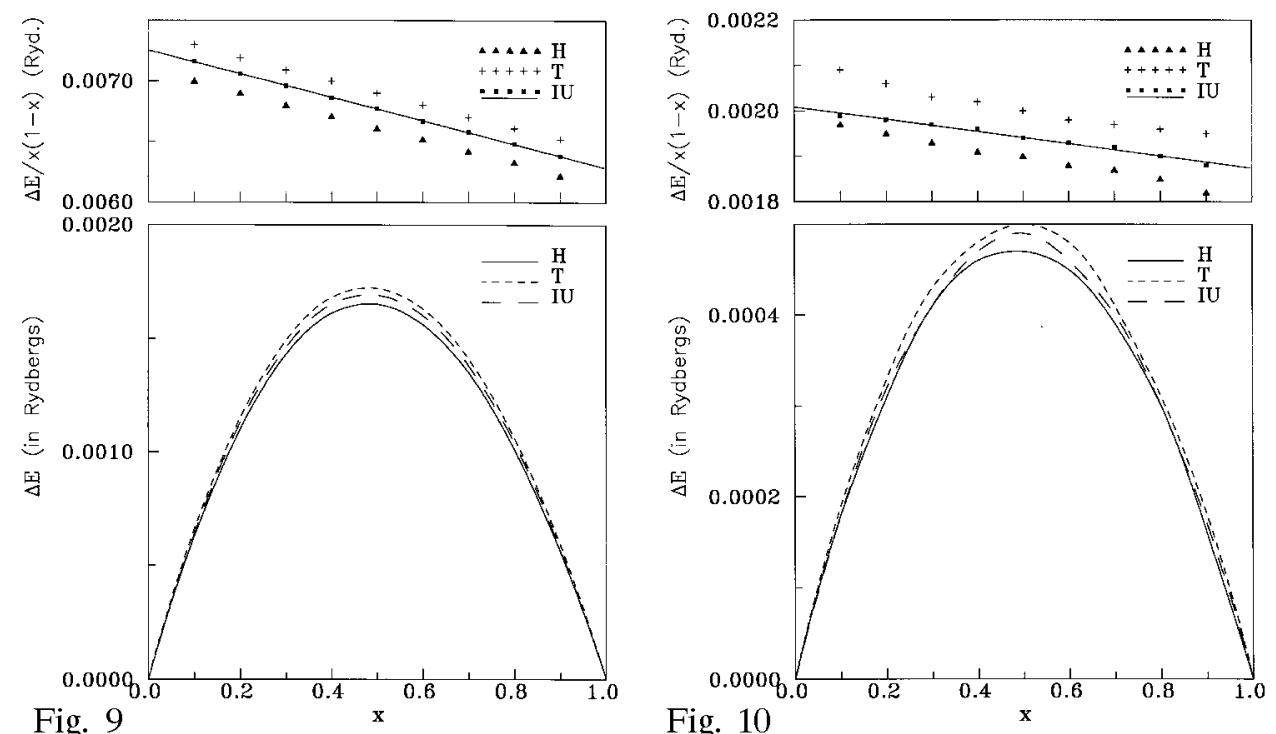

Fig. 9. The energy difference and interaction parameter for K-Cs alloy.

Fig. 10. The energy difference and interaction parameter for Rb-Cs alloy. 
The ratio of the heat of solution $\Delta E / x(1-x)$ for all the alkali systems shows a linear variation with atomic fraction. The lines show the best fitted one in the case of the IU screening function. The values due to the $\mathrm{H}$ and $\mathrm{T}$ screening functions also show a linear behaviour. The best fitted line in each case is expressed by (in Rydbergs)

$$
\begin{array}{ll}
\Delta E(x) / x(1-x)=-0.002125 x+0.014910, & \text { for LiNa system, } \\
\Delta E(x) / x(1-x)=-0.023706 x+0.073353, & \text { for LiK system, } \\
\Delta E(x) / x(1-x)=-0.039306 x+0.102830, & \text { for LiRb system, } \\
\Delta E(x) / x(1-x)=-0.065890 x+0.146013, & \text { for LiCs system, } \\
\Delta E(x) / x(1-x)=-0.003092 x+0.017591, & \text { for NaK system, } \\
\Delta E(x) / x(1-x)=-0.007267 x+0.030981, & \text { for NaRb system, } \\
\Delta E(x) / x(1-x)=-0.000135 x+0.002008, & \text { for RbCs system. } \\
\Delta E(x) / x(1-x)=-0.000968 x+0.007253, & \text { for KCs system, } \\
\Delta E(x) / x(1-x)=-0.000128 x+0.001508, & \text { for KRb system, }
\end{array}
$$

The first term in the fitted equations is very small indicating that the ratio of the heat of solution may be considered to be nearly a constant. It is difficult to carry out a detailed comparison without experimental studies on these alloys in the solid phase. Yokokawa and Kleppa [18] have measured the heat of solution in the liquid state of $\mathrm{K}-\mathrm{Rb}$ system at $111^{\circ} \mathrm{C}$ over the full composition range. They found that the ratio $\Delta E / x(1-x)$ does not depend on $x$. The present results have been obtained for the solid phase and cannot be compared directly with the results of the liquid phase, nevertheless it is found that in the solid phase all the alloy systems considered have given a value of $\Delta E / x(1-x)$ which is to be considered independent of $x$. 

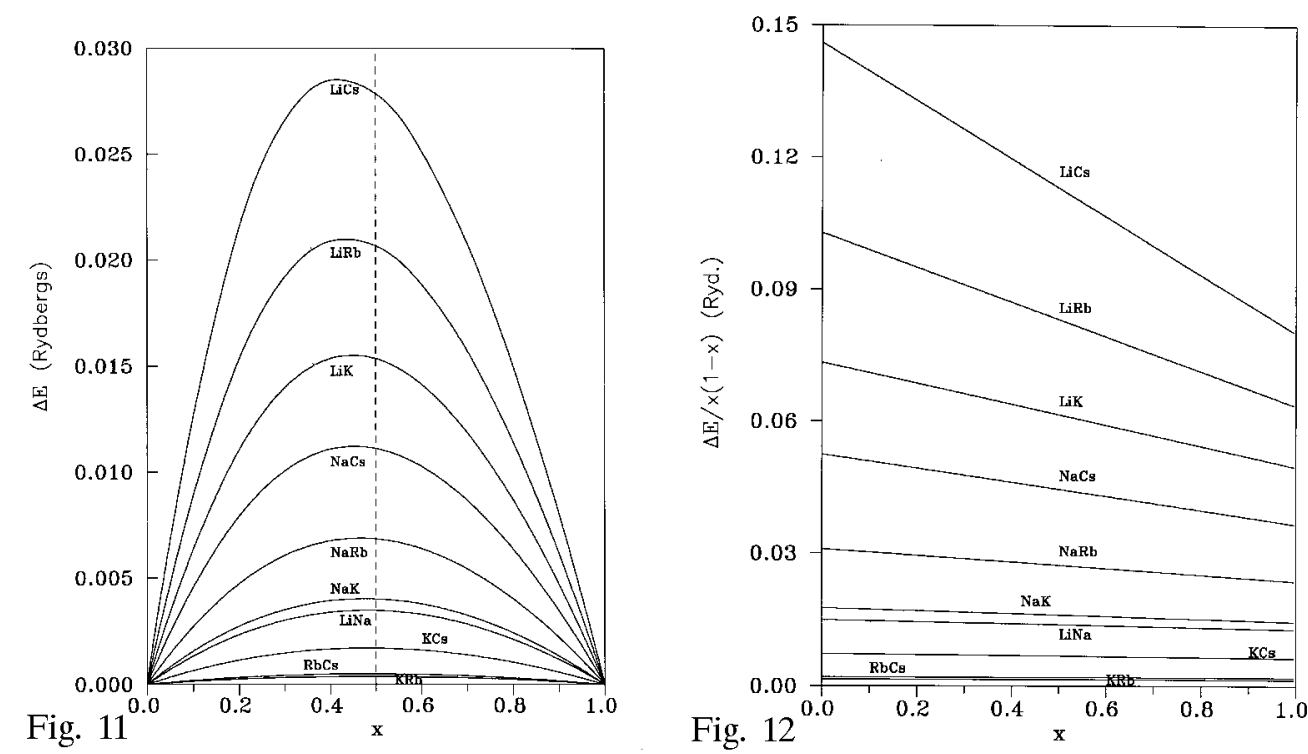

Fig. 11. The energy difference for binary alkali alloys using the IU function.

Fig. 12. The interaction parameter for binary alkali alloys using the IU function.

Figure 11 shows the energy difference for all the alkali based alloy systems found using the IU screening function while the ratio $\Delta E / x(1-x)$ is plotted against atomic concentration in Fig. 12.

Figure 11 shows that the curves representing the energy difference are not symmetrical about the concentration of 0.5 in all the cases. The asymmetry in the curves increases with the increase in the ratio of volumes of the two elements making up the alloy. However all the curves are seen to have $x$ dependence which can be fitted to polynomials. It is also seen that the numerical values of the energy difference increases with the increase in the ratio of the atomic sizes, the highest in the present study being that for the LiCs system. Higher values of energy difference show that solubility is less. For the Li based alloys as we go from $\mathrm{Na}$ towards $\mathrm{Cs}$, the metal having a smaller atomic size gives a smaller energy difference indicating a better solubility. A similar trend is observed for other combinations as well.

From Fig. 12 we can see that the ratio $\Delta E / x(1-x)$ is very nearly constant in the case of KRb, RbCs, KCs, LiNa, and $\mathrm{NaK}$ but where the ratios of the atomic sizes are large it is not seen to be a constant. The largest deviation from a constant for a ratio is predicted for $\mathrm{LiCs}$.

The present results obtained by our model potential in the PAA model are encouraging and may be further extended in the study of the lattice dynamics and the anharmonic properties of these systems. This will require further investigations. 


\section{Acknowledgment}

The author (P.N.G.) is thankful to the University Grants Commission, New Delhi, India, for providing the financial assistance.

\section{References}

[1] T.M. Hayes, H. Brookes, A. Stock, Phys. Rev. 175, 699 (1968).

[2] J.K. Inglesfield, J. Phys. C 1, 1337 (1968).

[3] D. Stroud, N.W. Ashcroft, J. Phys. F 1, 113 (1971).

[4] G.L. Krasko, Z.A. Gurskii, Sov. Phys. Solid State 13, 2062 (1972).

[5] P.N. Gajjar, B.Y. Thakore, A.R. Jani, Acta Phys. Pol. A 94, 33 (1998).

[6] B.Y. Thakore, Ph.D. Thesis, Sardar Patel University, Vallabh Vidyanagar (India) 1998.

[7] P.N. Gajjar, B.Y. Thakore, A.R. Jani, Proc. Solid State Phys. Symp., India C 40, 217 (1997).

[8] P.N. Gajjar, B.Y. Thakore, A.R. Jani, The Physics of Disordered Materials, NISCOM, New Delhi (India) 1997, p. 135.

[9] P.N. Gajjar, B.Y. Thakore, A.R. Jani, Proc. Solid State Phys. Symp., India C 38, 224 (1995).

[10] V.N. Patel, D.R. Mawani, B.Y. Thakore, P.N. Gajjar, A.R. Jani, Proc. Solid State Phys. Symp., India C 41, 233 (1998).

[11] J. Hafner, Phys. Rev. B 15, 617 (1977).

[12] S. Tanigawa, M. Doyama, Solid State Commun. 11, 787 (1972); J. Phys. F 2, L95 (1972); J. Phys. F 3, 977 (1973); Phys. Status Solidi B 56, 665 (1973).

[13] A.R. Jani, P.N. Gajjar, H.K. Patel, Phys. Status Solidi B 169, K105 (1992).

[14] P.N. Gajjar, B.Y. Thakore, J.S. Luhar, A.R. Jani, Acta. Phys. Pol. A 86, 369 (1994).

[15] T. Soma, H. Matsuo, Y. Kohw, Phys. Status Solidi B 107, 761 (1981).

[16] T. Soma, H. Matsuo, M. Funaki, Phys. Status Solidi B 108, 221 (1981).

[17] B.N. Tewari, K.N. Khanna, Physica B 123, 201 (1984).

[18] T. Yokokawa, O.J. Kleppa, J. Chem. Phys. 40, 46 (1964).

[19] W. Harrison, Pseudopotential in the Theory of Metals, W.A. Benjamin Pub. Co., New York 1966.

[20] R. Taylor, J. Phys. F 8, 1699 (1978).

[21] S. Ichimaru, K. Utsumi, Phys. Rev. B 6, 875 (1981).

[22] C. Kittle, Introduction to the Solid State Physics, 4th ed., Wiley Eastern Ltd., New Delhi 1966.

[23] Handbook of Chemistry and Physics, Ed. R.C. Weast, 56th ed., CRC Press, Cleaveland 1975. 